\title{
Advanced thermal lattice Boltzmann method for the simulation of latent heat thermal energy in a porous storage unit
}

\author{
Riheb Mabrouk ${ }^{1 *}$, Hassane Naji ${ }^{2}$, Hacen Dhahri ${ }^{1}$, Zohir Younsi ${ }^{2}$ \\ 1 University of Monastir, National Engineering School of Monastir, Thermal and Energy Systems Studies laboratory, Rue Ibn Jazza, \\ 5019 Monastir, Tunisia. \\ ${ }^{2}$ Univ. Artois, Univ. Lille, IMT Lille-Douai, Junia-HEI Hauts de France, Laboratoire Génie Civil \& géo-Environnement (ULR \\ 4515), Technoparc Futura, F-62400 Béthune, France.
}

\begin{abstract}
The current research expounds numerical investigation of key parameters effects, namely porosity ( $\varepsilon=0.4,0.6,0.7$ and 0.8$)$, Reynolds number $(R e=100,200,400$ and 600$)$ and Eckert number $(E c=0,1,5$ and 10) on the forced convective laminar flow and heat transfer through a horizontal porous channel filled with a metal foam structure impregnated with paraffin as a phase change material (PCM). The Darcy-Brinkman-Forchheimer model under the local thermal non-equilibrium (LTNE) condition is deemed at the representative elementary volume (REV) scale. The fully coupled equations of NavierStokes, Poisson's equation, energy equations, and continuity equation were handled numerically via a thermal single relaxation time lattice Boltzmann method (TSRT-LBM). To facilitate implementation, all LB equations are based on the same speed discretization scheme (D2Q9). Three-population distribution functions were applied to simulate the fluid flow, and temperatures of the fluid and solid phases. Previously, the numerical model was validated by available cases. Then, a comprehensive investigation has been performed to investigate the influence of the aforementioned dimensionless numbers. All LBM results are found to be highly consistent with other numerical works. The outcomes reported that at lower porosities, the energy and exergy efficiencies increased with increasing Re and Ec. However, for large porosity values, the efficiencies were optimum for a critical $\operatorname{Re} \sim 400$. To sum up, it can be stated that the implemented thermal lattice Boltzmann method has been demonstrated as a suitable approach study the thermal sensible energy storage.
\end{abstract}

\footnotetext{
*Correspondingauthor:mabroukriheb@gmail.com
} 


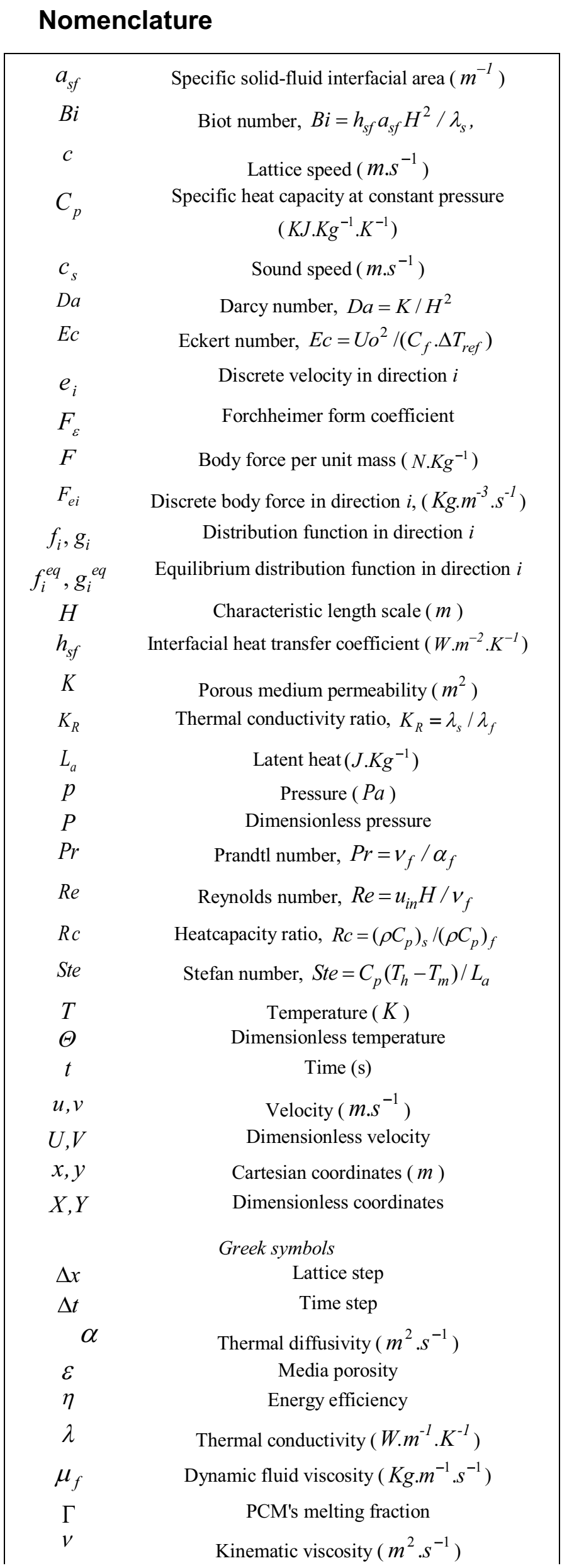

\begin{tabular}{cc|}
$\omega$ & Pore density (PPI) \\
$\psi$ & Exergy efficiency \\
$\rho$ & Density $\left(K g \cdot \mathrm{m}^{-3}\right)$ \\
$\tilde{t}$ & Dimensionless time \\
$\tau$ & Dimensionless relaxation time \\
$w_{i}$ & Weight coefficient in direction $i$ \\
& \\
$\mathrm{f}$ & Subscripts \\
$\mathrm{s}$ & Fluid \\
$\mathrm{h}$ & Solid \\
$\mathrm{m}$ & Hot \\
in & Melting \\
out & Inlet \\
Ref & Outlet \\
& Reference
\end{tabular}

\section{Introduction}

Among the various thermal energy storage (TES) systems, latent heat thermal energy storage (LHTES) systems have been considered one of the most popular solutions for saving energy. In a LHTES system, a substance called Phase Change Material (PCM) changes its state during melting and/or solidification. Owing to its small volume, low temperature fluctuation and high latent heat, thermal energy can be stored or released [1]. However, the low thermal conductivity of PCMs is the main drawback that slows down their charge and discharge times. To overcome this weakness, the highly conductive porous medium (metallic foam, copper, etc.) is one of the best techniques for improving thermal performance [2]. To this end, Yang et al. [3] proposed a structure of a porous metal foam to improve thermal energy storage via a PCM. In addition, numerical results revealed that a metallic foam with a modified porosity (linearly) could accelerate the melting time and improve the thermal performance compared to the case of uniform porosity. Ren et al. [4] dealt with a comparative study of the LHTES unit with different combinations of nanoparticles and metallic foams. Their findings demonstrated the effectiveness of metal foam. They stated that the decrease of the metal porosity speeds up the melting rate. Sardari et al. [2] conducted a study examining porosity and pore size effects of metal foams on a PCM's phase transition. They found that low porosity ensures high performance while speeding up the melting process by $85 \%$ compared to pure PCM. It is woth noting that the study of latent heat thermal energy in a porous storage unit can be handled experimentally and / or numerically. In this study, Boltzmann's lattice method (LBM) being an efficient numerical method due to its simplicity and ability to handle complex phenomena in porous media, was considered herein. Gao et al [5] have dealt with phase change phenomenon in porous structure coupled with conduction fins using the LBM at the representative elementary volume (REV) scale. Mabrouk et al. [6] successfully applied LBM at the REV scale to investigate the PCM melting behavior in metallic 
foams. Furthermore, Jourabian et al. [7] studied the melting cycle in a porous cavity numerically using an enthalpy-based LBM and showed the method's ability to address latent heat transfer. The follow-up structure of the study is as follows: In Section 2, basic equations, mathematical formulation along with assumptions are outlined. Then, in Section 3, the numerical approach (LBM) and validation are briefly exhibited. In Section 4 , the outcomes are discussed. Finally, the article wraps up by highlighting our main findings in section 5 .

\section{Problem statement}

\subsection{Geometry details}

The schematic diagram of the present computational domain is illustrated in Figure 1. The open straight channel of height $\mathrm{H}$ consists of upper and lower walls of length L, which are assumed to be adiabatic, impermeable and non-slip. Its height is considered as a characteristic dimension in the computations. The channel is fully filled with a porous metal foam and paraffin as a PCM embedded in its pores. The fluid enters the channel uniformly at a velocity $U_{\text {in }}$ and at a hot temperature $\mathrm{T}_{\mathrm{f}, \mathrm{h}}$ to initiate fusion. Note that the discharge process (return flow) is started with the velocity $-\mathrm{U}_{\text {in }}$ and a cold temperature $\mathrm{T}_{\mathrm{f}, \mathrm{c}}$.

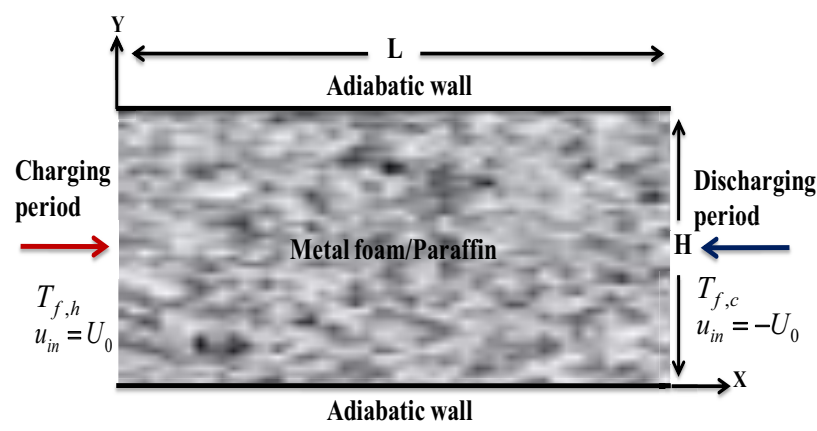

Fig. 1. Channel geometry

\subsection{Assumptions drawn up}

The mathematical system is based on the following hypotheses:

- Fluid flow and liquid paraffin are Newtonian, incompressible and laminar.

- Thermo-physical properties of porous matrix and PCM are constant, homogeneous and isotropic.

- Viscous dissipation is considered.

- LTNE condition is involved between solid and fluid phases.

\subsection{Governing equations}

The dimensionless governing equations for mass, momentum, and energy at the REV scale are adopted as follows [8-9]:

$\nabla \cdot \overrightarrow{\boldsymbol{U}}=0$

$$
\begin{aligned}
& \frac{\partial \vec{U}}{\partial \tilde{t}}+(\vec{U} \cdot \nabla)\left(\varepsilon^{-1} \vec{U}\right)=-\nabla(\varepsilon P)+R^{-1} \nabla^{2} \vec{U}+\varepsilon \cdot \vec{F} \\
& \frac{\partial \Theta_{f}}{\partial \tilde{t}}+U . \nabla \Theta_{f}=\frac{1}{\operatorname{Re} \cdot \operatorname{Pr}} \nabla \cdot\left(\frac{\lambda_{e f f, f}}{\lambda_{f}} \nabla\left(\frac{\Theta_{f}}{\varepsilon}\right)\right)+ \\
& K r \cdot \frac{B i}{\operatorname{Re} \cdot \operatorname{Pr}}\left(\frac{\Theta_{s}-\Theta_{f}}{\varepsilon}\right)-\frac{1}{S t e} \frac{\partial \Gamma}{\partial \tilde{t}}+\tilde{\Phi} \\
& \frac{\partial \Theta_{S}}{\partial \tilde{t}}=\frac{K r}{R c} \frac{1}{\operatorname{Re} \cdot \operatorname{Pr}} \nabla \cdot\left(\frac{\lambda_{\text {eff }, s}}{\lambda_{S}} \nabla\left(\frac{\Theta_{S}}{1-\varepsilon}\right)\right)- \\
& \frac{K r}{R c} \cdot \frac{B i}{\operatorname{Re} \cdot \operatorname{Pr}}\left(\frac{\Theta_{s}-\Theta_{f}}{1-\varepsilon}\right) \\
& \text { where, } \vec{F}=-\left(\frac{1}{\operatorname{ReDa}}+\frac{F_{\varepsilon}}{\sqrt{D a}}\|\vec{U}\|\right) \vec{U} \\
& \tilde{\Phi}=\varepsilon \cdot E c\left\{\frac{1}{D a \cdot R e}+\frac{F_{\varepsilon}}{\sqrt{D a}}\|\vec{U}\|\right\}\|\vec{U}\|^{2}+ \\
& \frac{E c}{\operatorname{Re}}\left\{2\left[\left(\frac{\partial \vec{U}}{\partial X}\right)^{2}+\left(\frac{\partial \vec{V}}{\partial Y}\right)^{2}\right]+\left(\frac{\partial \vec{U}}{\partial Y}+\frac{\partial \vec{V}}{\partial X}\right)^{2}\right\} \\
& \tilde{\Gamma}=\left\{\begin{array}{ll}
0 & \Theta<\Theta_{s} \\
\frac{\Theta-\Theta_{s}}{\Theta_{l}-\Theta_{s}} \text { if } & \Theta_{s} \leq \Theta \leq \Theta_{l} \\
1 & \Theta>\Theta_{l}
\end{array}\right\}
\end{aligned}
$$

The main normalization parameters deemed here are as follows:

$$
\begin{aligned}
& (X, Y)=(x, y) / H, U=u / U_{0}, P=p / \rho U_{0}^{2}, \tilde{t}=t U_{0} / H, \\
& \Theta=\left(T-T_{c}\right) /\left(T_{h}-T_{c}\right), \Delta T_{r e f}=T_{h}-T_{c}, D a=K / H^{2}, \\
& \operatorname{Pr}=v_{f} / \alpha_{f}, \operatorname{Re}=U_{i n} H / v_{f}, R c=\left(\rho C_{p}\right)_{s} /\left(\rho C_{p}\right)_{f}, \\
& K r=\lambda_{s} / \lambda_{f}, B i=h_{s f} a_{s f} H^{2} / \lambda_{s}, S t e=C_{p f}\left(T_{h}-T_{m}\right) / L a, \\
& E c=U_{0}^{2} /\left(C_{f} \Delta T_{r e f}\right)
\end{aligned}
$$

Here, $\overrightarrow{\boldsymbol{U}}$ and $\mathrm{P}$ are the volume-averaged velocity and the pressure. $\varepsilon$ and $\vec{F}$ denote the porous medium's porosity and the total force due to its presence and other external forces. $\Theta_{f / s}, \Gamma$ and $\tilde{\Phi}$ are the fluid/solid (porous medium) temperature, the PCM liquid fraction and the viscous dissipation, respectively.

\subsection{Energy and exergy efficiencies}

The overall energy $(\eta)$ and exergy $(\psi)$ efficiencies can be defined as follows [8]:

$$
\begin{aligned}
\eta & =\eta_{\text {charging }} \times \eta_{\text {discharging }}=100 \times\left(E_{\text {recovered }} / E_{\text {input }}\right) \\
\psi & =\psi_{\text {charg } \text { ing }} \times \psi_{\text {discharging }} \\
& =100 \times\left(\text { Ex }_{\text {recovered }} / \text { Ex } x_{\text {input }}\right)
\end{aligned}
$$




\subsection{Lattice Boltzmann equation (LBE)}

In this study, the single relaxation time-lattice Boltzmann (SRT-LBM) model was adopted to handle the propagation of fluid flow particles a lattice $\mathrm{x}$ at time $\mathrm{t}$ with finite discrete distribution velocities. Thereby, three distribution functions of the particle are involved for for the dynamic $\left(f_{i}(x, t)\right)$ and thermal ( $\left.g_{i, f, s}(x, t)\right)$ fields, the LTNE conjecture being assumed in this work $[8,10]$ :

$$
\begin{aligned}
& \underbrace{f_{i}\left(\vec{x}+\vec{e}_{i} \delta t, t+\vec{e}_{i} \delta t\right)-f_{i}(\vec{x}, t)}_{\text {strea } \min g}= \\
& -\delta t\left(f_{i}(\vec{x}, t)-f_{i}^{e q}(\vec{x}, t)\right) / \tau_{v}+\delta t . \quad \vec{F}_{e_{i}} \\
& \text { collision term } \\
& \text { force term } \\
& \underbrace{g_{f, i}\left(\vec{x}+\vec{e}_{i} \delta t, t+\delta t\right)-g_{f, i}(\vec{x}, t)}_{\text {strea min } g}= \\
& -\underbrace{\left(g_{f, i}(\vec{x}, t)-g_{f, i}^{e q}(\vec{x}, t)\right) / \tau_{T, f}}_{\text {collision term }} \\
& \underbrace{+\left(1+\delta t \partial_{t} / 2\right) \delta t S r_{i, f}+\delta t f_{i}(\vec{x}, t) q_{i}}_{\text {source terms }} \\
& \underbrace{g_{s, i}\left(\vec{x}+\vec{e}_{i} \delta t, t+\delta t\right)-g_{s, i}(\vec{x}, t)}_{\text {strea } \min g}= \\
& -\underbrace{\left(g_{S, i}(\vec{x}, t)-g_{S, i}^{\text {eq }}(\vec{x}, t)\right) / \tau_{T, S}}_{\text {collision term }} \\
& +\underbrace{\left(1+\delta t \partial_{t} / 2\right) \delta t S r_{i, s}}_{\text {source term }}
\end{aligned}
$$

Subscripts $f$ and $s$ point out the fluid and solid phases, respectively.

The local equilibrium distribution functions $f_{i}^{e q}(\vec{x}, t)$ and $g_{i, f ; s}^{e q}$ can be expressed under the D2Q9 lattice model as follows [10]:

$$
\begin{aligned}
& f_{i}^{e q}=\rho w_{i}\left(1+\frac{\vec{e}_{i} \cdot \vec{u}}{c_{s}^{2}}+\frac{\vec{u} \otimes \vec{u}:\left(\vec{e}_{i} \otimes \vec{e}_{i}-c_{s}^{2} I\right)}{2 c_{s}^{4} \varepsilon}\right) \\
& g_{f, i}^{e q}=w_{i} T_{f}\left(1+\vec{e}_{i} u /\left(\varepsilon c_{s}^{2}\right)\right) \text { and } g_{s, i}^{e q}=w_{i} T_{s}
\end{aligned}
$$

The last terms in Eq. (11)- (13) are computed as follows [8]:

$$
\begin{aligned}
& \overrightarrow{F_{e_{i}}}=w_{i} \rho\left(1-\frac{1}{2 \tau_{v}}\right)\left[\frac{\vec{e}_{i} \cdot \vec{F}}{c_{s}^{2}}+\frac{\vec{u} \cdot \vec{F}:\left(\vec{e}_{i} \vec{e}_{i}-c_{s}^{2} I\right)}{\varepsilon c_{s}^{4}}\right] \\
& S r_{i, f}=w_{i}\left(\frac{L a}{C_{p, f}}\left[\frac{\gamma(t+\delta t)-\gamma(t)}{\delta t}\right]+\frac{h\left(T_{s}-T_{f}\right)}{\varepsilon\left(\rho C_{p}\right)_{f}}\right)
\end{aligned}
$$

$$
\begin{aligned}
& S r_{i, s}=w_{i}\left(\frac{h\left(T_{s}-T_{f}\right)}{(1-\varepsilon)\left(\rho C_{p}\right)_{s}}\right) \\
& q_{i}=-\left(f_{i}-f_{i}^{e q}\right)\left(\vec{e}_{i}-\vec{u}\right)\left(\vec{e}_{i}-\vec{u}\right): \Delta \vec{u}
\end{aligned}
$$

Finally, $\rho, \vec{u}, T_{f}, T_{s}$ can be written as:

$$
\begin{aligned}
& \rho=\sum_{i} f_{i}, \quad \rho \vec{u}=\sum_{i} f_{i} \vec{e}_{i}+\delta t \vec{F} / 2, \\
& T_{f}=\sum g_{f i} \text { and } T_{s}=\sum g_{s i}
\end{aligned}
$$

\subsection{Code validation}

To validate our in-house code, figure. 3 presents the comparison of the difference between temperatures with results of Abdedou et al. [11] at two axial positions $X=0.14$ and $X=0.5$.

Through these comparisons, it can be stated that our in-house code based on the LB approach denotes satisfactory results.

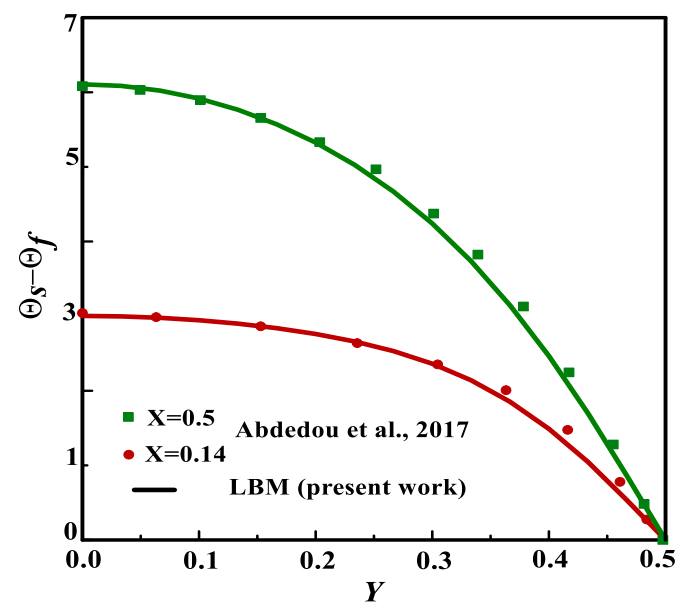

Fig. 2. $\Theta_{s}-\Theta_{f}$ vs. dimensionless transverse distance for $D a=10^{-4}, B i=0.01, R e=100=R q$ and $K r=1$.

\section{Results and comments}

It is worth recalling that the forced melting/solidification of paraffin under the LTNE assumption in an open porous channel, meshed in $480 \times 120$ cells, is here simulated using a thermal SRTLBM.

First, note that the LTNE assumption is secured using the following criterion [12]:

$$
L T N E=\sum_{N}\left|\Theta_{s}-\Theta_{f}\right| / N
$$

Figure 3 exemplifies the validity of the LTNE assumption vs. the interstitial heat transfer coefficient ( $h_{s f}$ ) for Re numbers and porosities considered. It is found that the hypotheses LTNE and LTE depend on (Reynolds number). It may be pointed out that, for the lowest porosity value ( $\varepsilon=0.4$ ), as Re increases (from 
100 to 400), $h_{s f}$ increases from 219 to 1200 (which corresponds to $B i$ from 0.3 to 1.62 ) indicating that the LTE holds indicating that the LTE holds. However, for $\mathrm{Re}=600$, it is the LTNE which holds for all the $h_{s f}$ ( $B i$ ) values except for high $h_{s f} \geq 6650(B i \geq 9)$. As soon as the porosity increases $(\varepsilon \geq 0.6)$, it is the LTNE which takes place for very small $h_{s f}$ (or $B i$ ) or even moderate and gives away to the LTE for high $h_{s f}(\geq 1650$ ) (or $B i \geq 2.2$ ). To sum up, one can state that the LTE is perfectly secured in the porous domain for interstitial heat transfer coefficient high values while being affected by the Re number. Simply put, and within the framework of our study, the system is in a non-equilibrium state for lower values of $h_{s f}$ (low $B i$ ). This finding is supported by other works [12], to name a few.

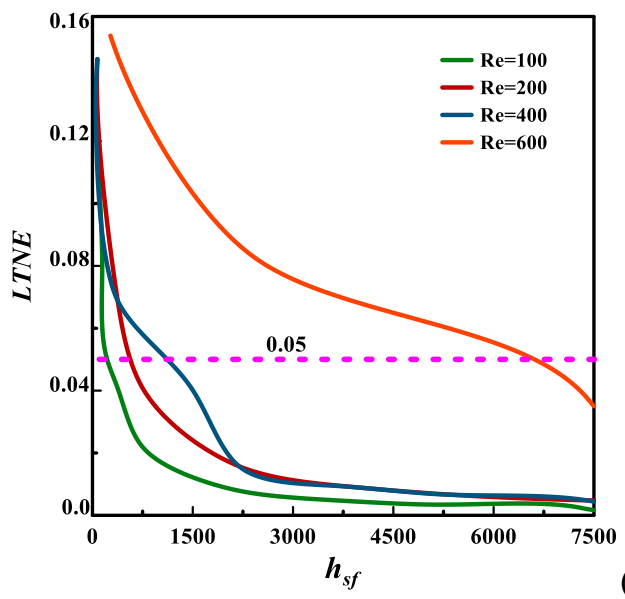

(a) $\varepsilon=0.4$

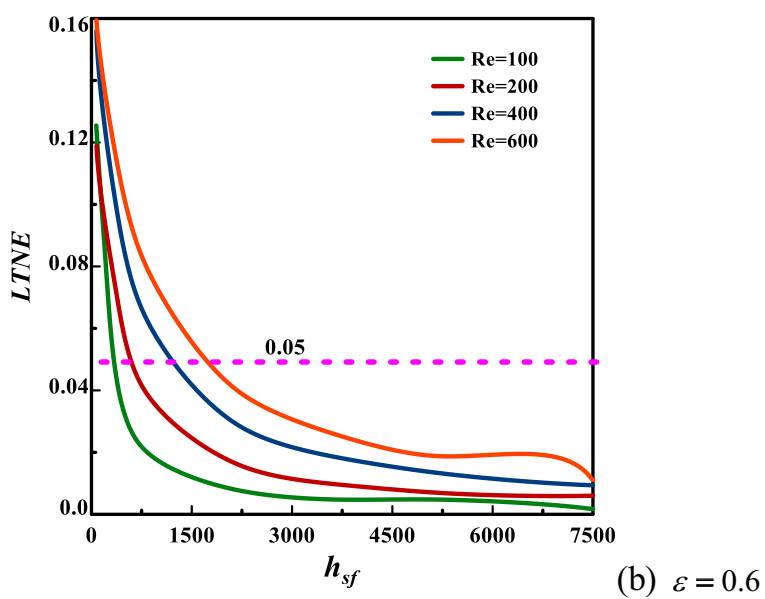

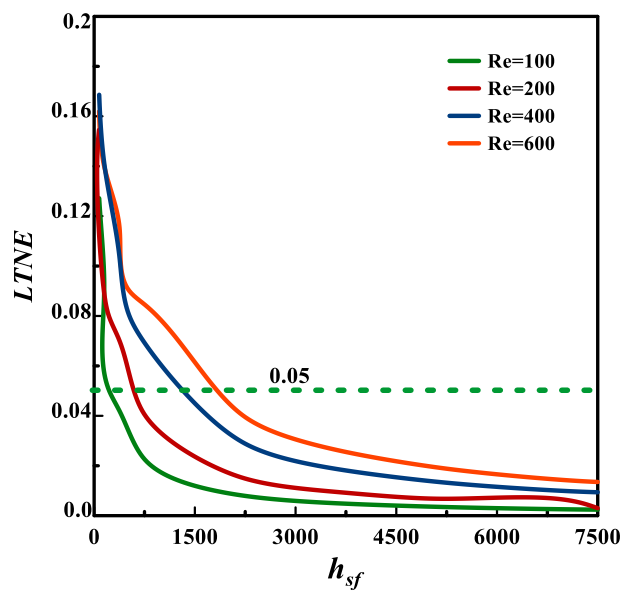

(c) $\varepsilon=0.7$

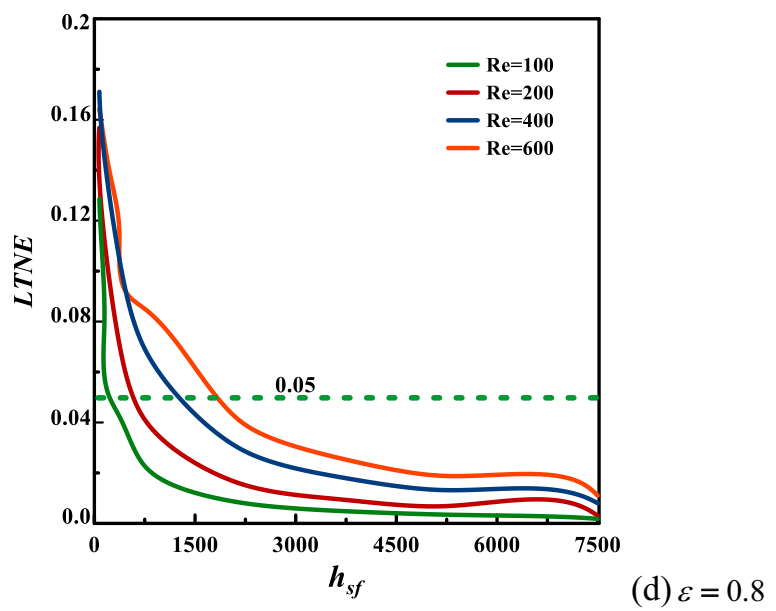

Fig. 3. LTNE vs. interfacial heat transfer coefficient ( $\left.h_{s f}\right)$ for four $\operatorname{Re}$ numbers with $\operatorname{Pr}=50$, Ste $=1, D a=10^{-3}, K r=1000, R c=1, E c=0$.

Figure 4 depicts the global energetic efficiency $(\eta)$ vs. $\mathrm{Re}$ for different Ec and $\varepsilon$ during the charging/discharging period. From this figure, it can be stated that $\eta$ increases very greatly with Re for small porosity values $(=0.4-0.6)$ whereas for large porosity (=0.7-0.8), $\eta$ reaches a maximum for $\operatorname{Re} \sqcup 400$, and then decreases. This involves that during the charging and discharging processes, the heat transfer intensifies owing to the Re increase, thereby rising the stored energy. It can also be seen that the global energetic efficiency is optimal for a critical Re value dependently of the porosity and Ec number. Thus, at larger porosity (=0.7-0.8), the energy stored is maximum for $\mathrm{Re}$ around 400 whereas paraffins stores the maximum of energy for $\mathrm{Re}=600$ at lower porosity. Moreover, it appears that the the energy efficiency increases when the porosity decreases owing to the low thermal conductivity of the PCM compared to the metal foam which leads to the weakness of the interfacial heat transfer between the solid and liquid phases. In addition, as Ec number increases, $\eta$ rises which means that the viscous forces affected strongly the energy stored in the system. 


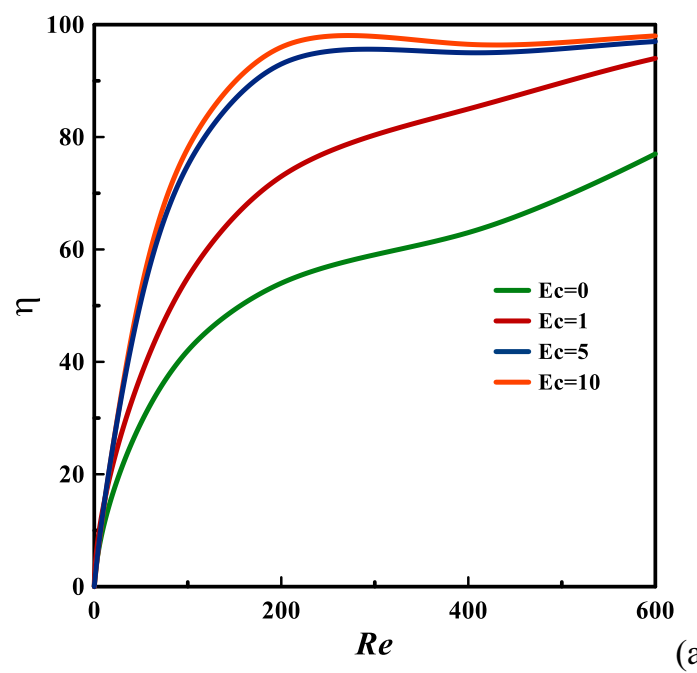

$\varepsilon=0.4$

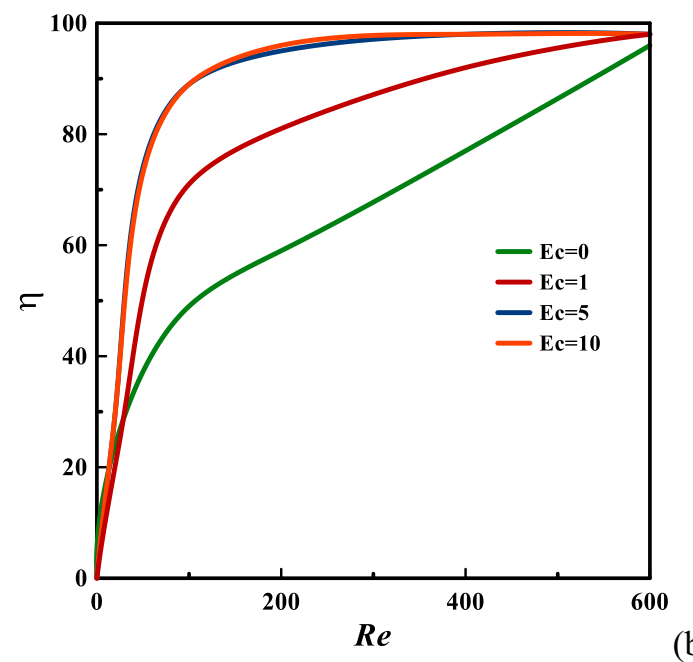

$\varepsilon=0.6$

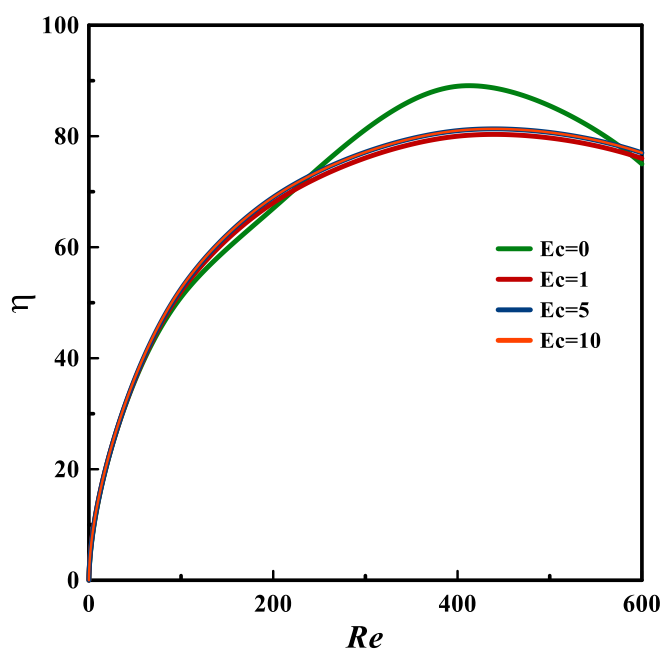

(c)

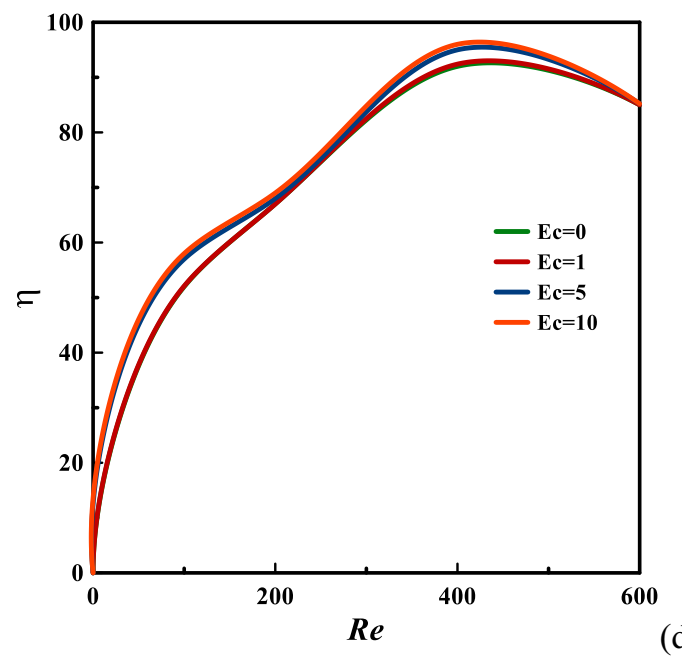

$\varepsilon=0.8$

(d)

Fig. 4. Energetic efficiency $(\eta)$ vs. Re for different Ec numbers with $\operatorname{Pr}=50, R c=1, B i=0.1$ and $S t e=1$.

Figure 5 depicts the global exergetic efficiency $(\psi)$ vs. Re for different Ec and $\varepsilon$ during charging/discharging period. As seen Figure 6 (a) and (b), at low porosity values $(=0.4-0.6)$, the global exergetic efficiency augments proportionally with $\mathrm{Re}$ and Ec. This is because the convective heat transfer and the body forces enhance the heat exchange between the two phases. However, for a large porosity $(=0.7-0.8)$, the global exergetic efficiency rises to reach its maximum for Re about 400 and then drops. It is worth noting that Ec effects are negligible for $\varepsilon=0.7-0.8$.

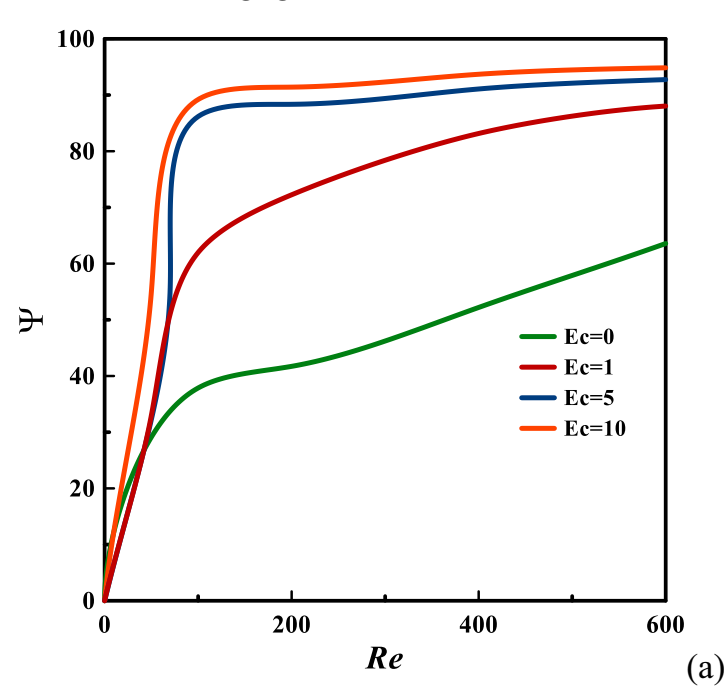

$\varepsilon=0.4$ 


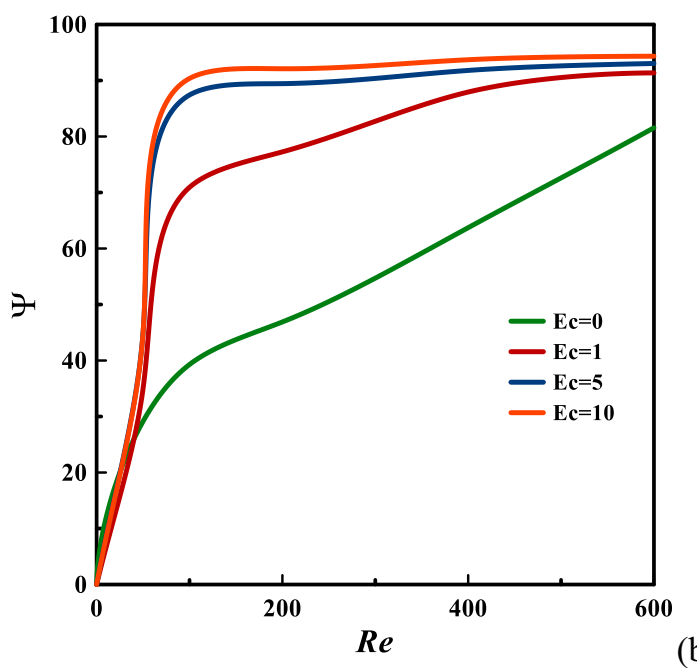

$\varepsilon=0.6$

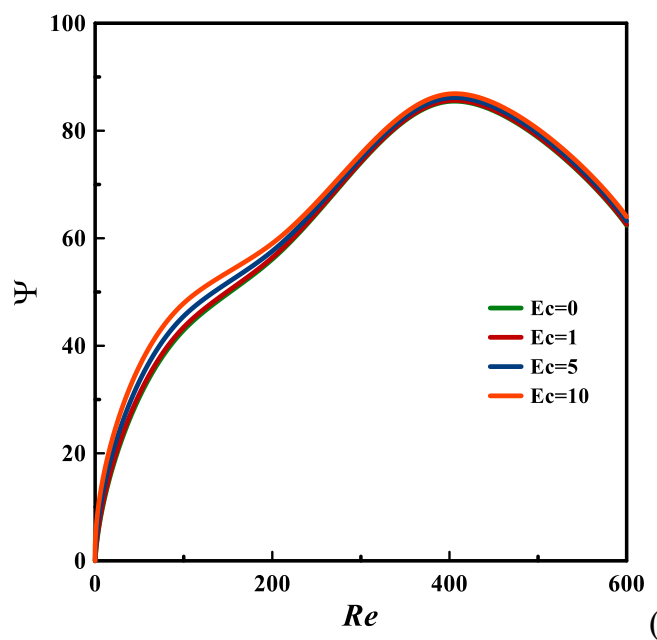

(c)

$\varepsilon=0.7$

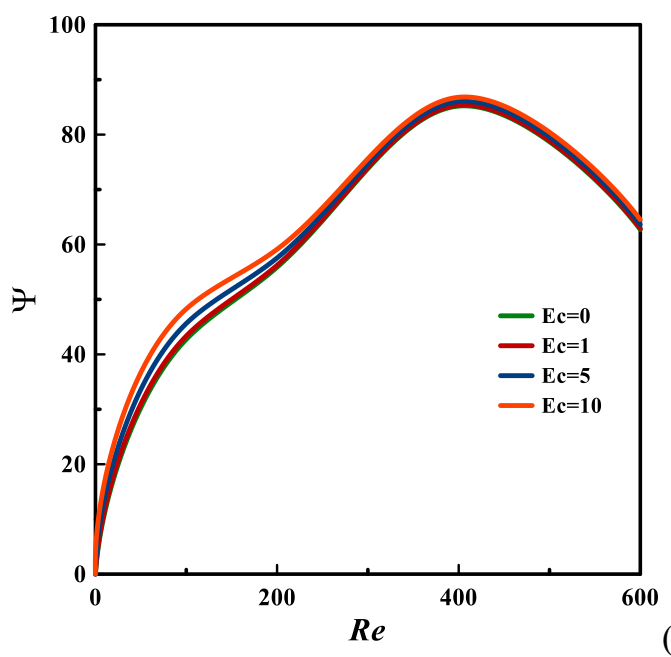

(d)

$\varepsilon=0.8$

Fig. 5. Exergetic efficiency $(\psi)$ vs. Re for different Ec numbers with $\operatorname{Pr}=50, R c=1, B i=0.1$ and $S t e=1$.

\section{Conclusions}

This two-dimensional numerical simulation is reported to primarily examine the effects of porosity, Reynolds number and Eckert number on forced laminar convection involved in a porous channel impregnated with paraffin.

From the obtained findings, the following conclusions can be brought forward:

At small porosity and high Re number, the LTNE intensity is important.

At high porosity values $(=0.7-0.8)$, the quantity of the stored energy is lower whatever Re and Ec.

At low porosity' values $(=0.4-0.6)$, the energetic and exergetic efficiencies rise proportionally with $\mathrm{Re}$ and Ec numbers.

\section{Acknowledgments}

The first author is currently completing her PhD. In addition, she did advanced internships at LGCG (ULR 4515)/University of Artois to perform numerical simulations under the supervision of Prof. H. NAJI. The authors express their gratitude to the universities and institutes concerned for their assistance of any kind (scholarships and others).

\section{References}

1. M.M. Farid, A.M. Khudhair, S. A.K. Razack, S. Al-Hallaj, Energy Conv. Manage 45, 1597-1615 (2004).

2. P.T. Sardari, H.I. Mohammed, D. Giddings, M. Gillott, D. Grant, Energy, 189, 116108 (2019).

3. J.L. Yang, L.J. Yang, C. Xu, X.Z. Du, Int. J. Heat Mass Transf., 84, 1008-1018 (2015).

4. Q. Ren, F. Meng, P. Guo, Int. J. Heat Mass Transf., 121, 1214-1228 ( 2018).

5. D. Gao, Z. Chen, D. Zhang, L. Chen, Appl. Therm. Eng. 118, 315-327 (2017).

6. R. Mabrouk, H. Naji, H. Dhahri, Z. Younsi, Energies, 13(15), 3979 ( 2020).

7. M. Jourabian, A. A. R. Darzi, D. Toghraie, O. ali Akbari, Physica A, 509, 316-335 (2018).

8. R. Mabrouk, H. Dhahri, H. Naji, S. Hammouda, Z. Younsi, Int. J. Heat Mass Transf., 153, 119606 (2020).

9. G.F. Al-Sumaily, M.C. Thompson, Int. J. Heat Mass Transf., 61, 226-244 (2013).

10. Y. B. Tao, Y. You, Y. L. He, Appl. Therm. Eng., 93, 476-485 (2016).

11. A. Abdedou, K. Bouhadef, R. Bennacer, Therm. Sci 21 (6A), 2413-2423, 2017.

12. G. F. Al-Sumaily, J. Sheridan, M.C. Thompson, Int. Commun. Heat Mass Trans 43, 30-38, (2013). 